\title{
Improving the productivity of okra (Abelmoschus esculentus L.) by strengthening the impact of applied nutrients through alligator weed compost
}

\author{
Asif Tanveer ${ }^{1}$, Muhammad Shahbaz Asghar ${ }^{1}$, Muhammad Sarwar ${ }^{1, *}$, Muhammad Farrukh Saleem ${ }^{1}$, \\ Mubashar Nadeem ${ }^{1}$, Muhammad Kashif Munir ${ }^{2}$, Muhammad Zafar ${ }^{2}$, Muhammad Rizwan ${ }^{3}$ and Ghulam \\ Sarwar $^{4}$ \\ ${ }^{1}$ Department of Agronomy, University of Agriculture Faisalabad, Pakistan; ${ }^{2}$ Agronomic Research Institute, Ayub \\ Agricultural Research Institute Faisalabad, Pakistan; ${ }^{3}$ PirMehr Ali Shah Arid Agriculture University, Rawalpindi, Pakistan; \\ ${ }^{4}$ Cotton Research Station, Ayub Agricultural Research Institute, Faisalabad, Pakistan \\ *Corresponding author’s e-mail: dr.sarwar@uaf.edu.pk; sarwar1406@gmail.com
}

\begin{abstract}
Weeds produce huge biomass by competing with the main crop for resources has been well established by numerous investigators. The use of weeds, especially aquatic weeds, as an organic source of nutrients such as compost is lacking in the literature. We hypothesized that the use of aquatic weeds i.e. alligator as compost would limit the use of inorganic fertilizers in okra and it would increase the quality of produce. To maximize the effect of inorganic nutrients, it would be easier to use the alligator weed compost as a natural source of nutrients. The crop was sown on 22 July, 2017 and 20 July, 2018 in a randomized complete block design having four replications. The net plot size was $1.8 \mathrm{~m}$ x $6 \mathrm{~m}$. The alligator weed compost along with NPK was applied at the time of sowing. Alligator weed compost was applied along with different combinations of NPK i.e. control (recommended dose of NPK 159-114-93 kg ha-1), compost application of $250 \mathrm{~kg} \mathrm{ha}^{-1}$, compost application of $500 \mathrm{~kg} \mathrm{ha}^{-1}$, compost application of $250 \mathrm{~kg} \mathrm{ha}^{-1}$ with $75 \%$ recommended NPK, compost application of $250 \mathrm{~kg} \mathrm{ha}^{-1}$ with $50 \%$ recommended NPK, compost application of $250 \mathrm{~kg} \mathrm{ha}^{-1}$ with $25 \%$ recommended NPK, compost application of $500 \mathrm{~kg} \mathrm{ha}^{-1}$ compost with $75 \%$ recommended NPK, compost application of $500 \mathrm{~kg} \mathrm{ha}^{-1}$ with $50 \%$ recommended NPK and compost application of $500 \mathrm{~kg} \mathrm{ha}^{-1}$ with $25 \%$ recommended NPK. Compost application of $250 \mathrm{~kg} \mathrm{ha}^{-1}$ with $50 \%$ recommended NPK significantly reduced the number of days to flowering and increased plant height $(\mathrm{cm})$ at flowering and plant maturity over the alone NPK and other combinations of compost and NPK. Pod yield and yield components were outclassed by the compost application of $250 \mathrm{~kg} \mathrm{ha}^{-1}$ with $50 \%$ recommended NPK over the other combinations. For example, number of pods per plant and pod yield $\left(\mathrm{t} \mathrm{ha}^{-1}\right)$ were increased by $26 \%, 20 \%$, respectively averaged across during both years of study over the recommended dose of NPK alone. Similarly, application of compost at $500 \mathrm{~kg} \mathrm{ha}^{-1}$ along with NPK $50 \%$ of recommended dose enhanced pod potassium and ascorbic acid contents by $76 \%$ and $35 \%$, respectively averaged across during both years of study over the control dose of NPK. Conclusively, alligator weed organic source of nutrients could reduce NPK fertilizers as it holds the nutrients applied and also provides the essential nutrients. In future climates, alligator weed compost would be a sustainable and environment friendly approach.
\end{abstract}

Keywords: Aquatic weed, curing, okra, nutrients re-use, quality, yield.

Abbreviations: BH Biomass harvested, Cd lead, DAE Days after emergence, DAS days after sowing, K potassium, N nitrogen, $\mathrm{Pb}$ cadmium, $\mathrm{PH}$ plant height, $\mathrm{P}$ phosphorous, $\mathrm{Zn}$ zinc.

\section{INTRODUCTION}

Aquatic plants disperse into the environment when these are introduced as ornamental plants into the country (Hansson and Fredriksson, 2004; Fortuna et al., 2005). Alligator weed (Alternanthera philoxeroides L.) is a terrible weed in the world and Pakistan that grows in water and land, impacting both rivers as well as flood plains. It is a perennial natural herb that adjusts to a series of soil types. It can grow successfully in both marine and terrestrial settings in tropical, subtropical and temperate areas (Julien et al., 1995). Weeds can be utilized as composting products, without losing them. But an extremely little information is available on the use of these plants for useful objectives. There is a need to focus on the

Asif, T., M.S. Asghar, M. Sarwar, M.F. Saleem, M. Nadeem, M.K. Munir, M. Zafar, M. Rizwan and G. Sarwar. 2021. Improving the productivity of okra (Abelmoschus esculentus L.) by strengthening the impact of applied nutrients through alligator weed compost. Pak. J. Agri. Sci. 58:1131-1139. [Received 16 Nov 2021; Accepted 26 Jul 2021; Published (online) 21 Sep 2021] 
utilization of weeds in production methods to ensure that benefits might gain from this aspect that has been greatly overlooked. Soil fertility is low in sub-tropical areas due to continuous cultivation. Sustainable crop production is possible through the use of compost, which increases organic matter within soil, improves soil physical structure and reduces the use of synthetic fertilizers (Smiciklas et al., 2008; Brown and Cotton, 2011). Compost is a cost-effective source of nutrients that can improve the soil fertility and organic matter of Pakistani soils (Sarwar, 2005). Organic fertilizers along with inorganic fertilizers can fulfill the nutrient requirement of okra, which is a good source of carbohydrates, protein, fat, minerals and vitamins (Savello et al., 1980). Okra plant requires plant nutrients for proper growth. Growth, yield and yield contributing attributes have been increased in okra by the use of manures, indicating that organic source of nutrients improve economical yield by providing essential nutrients for a longer period (Sameera et al., 2005; Tiamiyu et al., 2012). Integrated nutrient management is a good way to maintain soil fertility to an optimum level of crop production to maximize the benefits of all available plant nutrient sources (inorganic as well as organic) (Aulakh and Grant, 2008). Combinations of inorganic and organic fertilizers in soil have been used to increase okra production (Akande et al., 2010; Oyewole et al., 2011). It maximizes the use of available organic products and minimizes the use of expensive inorganic fertilizers purchased (Manral and Saxena, 2003).

There is little information available on the use of alligator weed as compost alone and with inorganic fertilizers as an integrated solution under field conditions. First, we hypothesized that alligator weed compost would be a good source of nutrients and would improve soil health. Second, the use of alligator weed compost would potentially improve the growth, yield and quality of okra by providing the essential nutrients that would minimize the use of inorganic nutrients, consequently would be a better approach for sustainable nutrient management. Considering the importance of compost, especially weed compost, and its role in integrated nutrient management, the present study was conducted with the objectives 1) to exploit the potential of Alligator weed as compost 2) to test the function of Alligator weed compost for nutrient supply and to verify the role of this weed compost with inorganic fertilizers.

\section{MATERIALS AND METHODS}

Experimental site: The experiment was conducted at Agronomic Research Farm, Department of Agronomy, University of Agriculture, Faisalabad Pakistan.

Compost preparation: Alligator weed biomass was harvested 45, 60 and 75 days after emergence, but biomass harvested 60 days after the emergence was used for compost preparation. Biomass was collected from the Farm Area, Department of
Agronomy, University of Agriculture, Faisalabad, Pakistan. Collected biomass was air-dried, sliced, then oven-dried at $70^{\circ} \mathrm{C}$ for 24 hours to remove excess moisture and finally crushed in the crushing unit of the compost to turn the raw form of biomass into finer form ( $<2 \mathrm{~mm}$ particles) to increase the surface area. The crushed material was moved to a compost (processing unit) with an appropriate level of moisture and an oxygen inlet, which provided aeration for the process of decomposition. Compost was ready after 20 days under controlled temperature, moisture and aeration.

Alligator weed compost analysis: Physio-chemical analysis of compost (Alligator weed biomass) harvested at 45, 60 and 75 days after emergence was carried out as described by Ryan et al. (2007) with findings as depicts in Table 1. The analysis was done at 45, 60 and 75 days after emergence to check after how many days after emergence the maximum nutrients were present in alligator weed biomass. The biomass harvested after 60 days of emergence had maximum nutrients and was used for compost preparation.

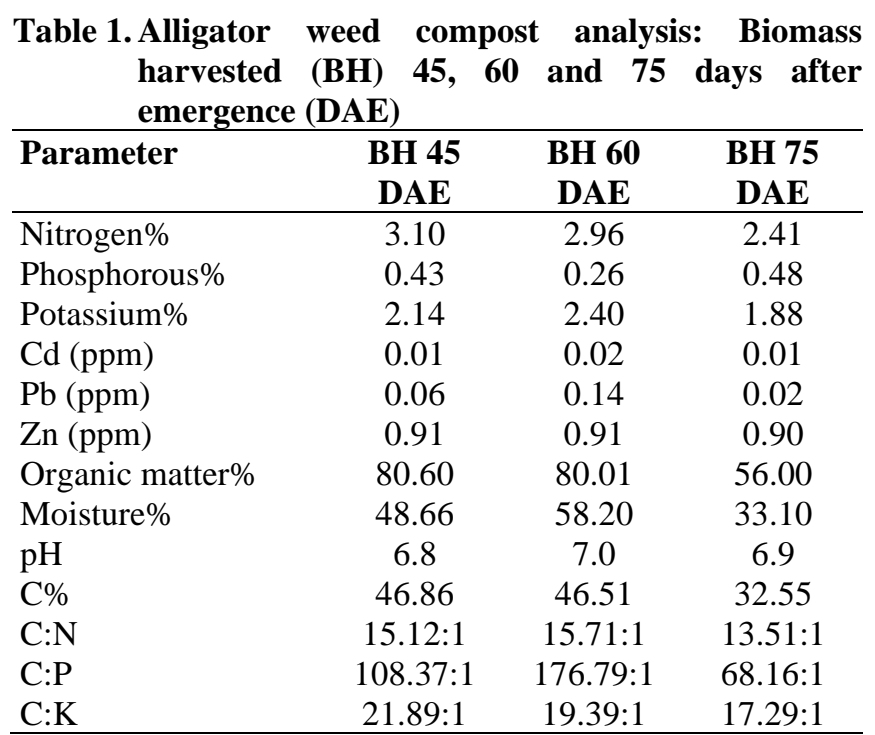

$\mathrm{BH}=$ Biomass harvested, DAE $=$ Days after emergence

Treatments: The experiment was comprised of nine treatments i.e. control (recommended dose of NPK 159-114$93 \mathrm{~kg} \mathrm{ha}^{-1}$ ), $250 \mathrm{~kg} \mathrm{ha}^{-1}$ compost, $500 \mathrm{~kg} \mathrm{ha}^{-1}$ compost, $250 \mathrm{~kg}$ $\mathrm{ha}^{-1}$ compost+ $75 \%$ recommended NPK, $250 \mathrm{~kg} \mathrm{ha}{ }^{-1}$ compost+ $50 \%$ recommended NPK, $250 \mathrm{~kg} \mathrm{ha}^{-1}$ compost+ $25 \%$ recommended NPK, $500 \mathrm{~kg} \mathrm{ha}^{-1}$ compost+ $75 \%$ recommended NPK, $500 \mathrm{~kg} \mathrm{ha}^{-1}$ compost+ $50 \%$ recommended NPK and $500 \mathrm{~kg} \mathrm{ha}^{-1}$ compost+ $25 \%$ recommended NPK. Before sowing, recommended dose of alligator weed compost was incorporated in soil according to the treatments.

Crop husbandry and sowing strategy: The crop was sown on 22 July, 2017 and 20 July, 2018. Well drained soil was selected to exclude any of the growth limitations either 
subject to waterlogging, salinity or sodicity. The soil was manipulated and pulverized with two cultivations followed by planking. Okra variety OH-152 was sown with the help of dibbler at $60 \mathrm{~cm}$ ridge to ridge distance. Plant to plant distance was maintained at $15 \mathrm{~cm}$ apart. The crop was sown in a randomized complete block design having four replications. The net plot size was $1.8 \mathrm{~m} \times 6 \mathrm{~m}$. Ridges were made manually with the help of spade. The crop was irrigated only with canal water. Insecticides were used to avoid crop damage from insects. All other practices were according to the respective experiment. The weather data during the whole crop growth period is given in Fig. 1.

(a)

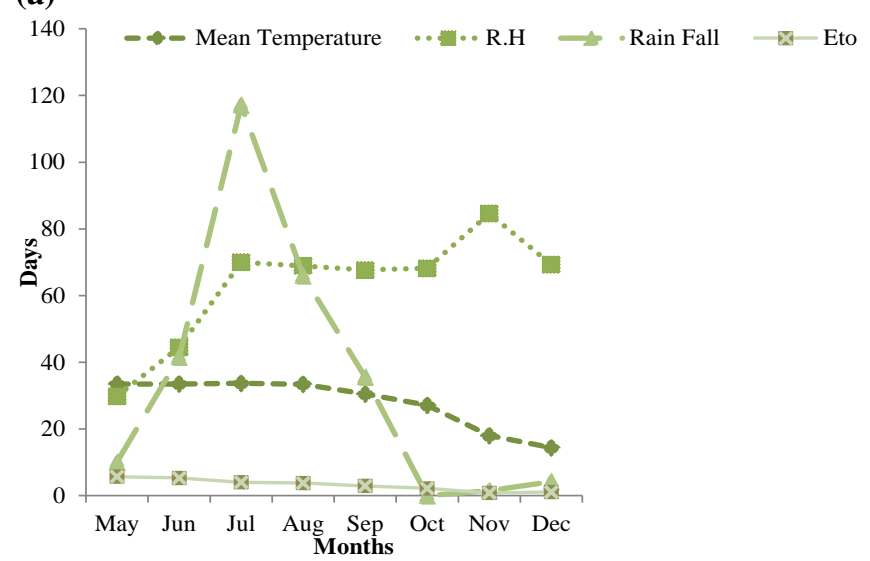

(b)

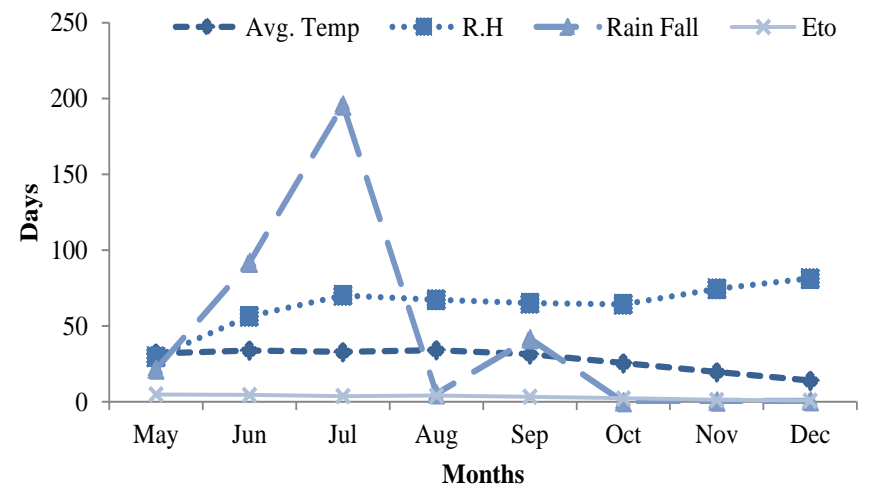

Figure 1. Weather conditions during the whole crop growth period (a) 2017 b) 2018.

Soil: Sandy loam textured fertile tract with six months' preplantation fallow history was selected and engaged for okra sowing purpose. Soil samples were collected from $15 \mathrm{~cm}$ depth before sowing of crop and were subjected to chemical analysis. Soil analysis was carried out with findings as depicts in Table 2.

Irrigation: Okra crop was irrigated keeping in view the conditions of the crop as observed visually by leaves of the crop. Plants under went light irrigations however, usual and unusual patterns of rainfall added to applied irrigation water.
Total nine irrigations were applied during the cropping period each after $6,13,20,33,40,60,67$ and 74 days of sowing.

Table 2. Physio-chemical analysis of soil

\begin{tabular}{lccc}
\hline Characteristics & $\begin{array}{c}\text { Unit } \\
\text { A) Depth of sample }\end{array}$ & $\begin{array}{c}\text { Value } \\
\mathbf{0 - 6}\end{array}$ & $\begin{array}{c}\text { Value } \\
\mathbf{6 - 1 2}\end{array}$ \\
\hline B) Mechanical analysis & & & \\
Sand & $\%$ & 52 & 52 \\
Silt & $\%$ & 20 & 20 \\
Clay & $\%$ & 28 & 28 \\
Textural class & & Sandy loam & \\
C) Chemical analysis & & & \\
Saturation & $\%$ & 32 & 32 \\
EC & dS/m & 1.6 & 1.4 \\
pH & -- & 7.8 & 7.8 \\
Organic matter & $\%$ & 0.35 & 0.25 \\
Total nitrogen & $\%$ & 0.028 & 0.022 \\
Available phosphorus & ppm & 7.9 & 8.9 \\
Available potassium & ppm & 200 & 220 \\
Available zinc & ppm & 1.1 & 1.3 \\
Available boron & ppm & 0.6 & 0.8 \\
\hline Total nitrogen, i.e. organic N+ inorganic N &
\end{tabular}

Fertilizers: Nitrogen fertilizer was applied through sidedressing manually by placing the fertilizer on side of the furrow near the plants except for the basal dose which was broadcasted and incorporated at seedbed preparation. Split doses (each dose was $1 / 4^{\text {th }}$ of the total amount) of $\mathrm{N}$ fertilizer were applied at four different stages i.e. at sowing, vegetative (33 days after sowing), flowering (54 days after sowing) and fruit formation. The whole of $\mathrm{P}$ was broadcasted and incorporated at seedbed preparation while half $\mathrm{K}$ was applied at sowing and the remaining half at fruit formation.

Insect Pests management: Insects that were observed during the crop period were whitefly (Bemisiatabaci), aphid (Aphis gossypii), okra fruit borer (Helicoverpaarmigera), thrip (Thripidae) and Leaf hopper (Amrascabiguttula). Insecticides i.e. MATCH 50EC at370 ml ha ${ }^{-1}$ (Lufenuron @ 50g a.i. L ${ }^{-1}$ ) and ACTARA 25WG at59.30 $\mathrm{g} \mathrm{ha}^{-1}$ (Thiamethoxamat $250 \mathrm{~g}$ a.i. $\mathrm{kg}^{-1}$ ) were used to control damage by these insects. These insecticides were bought from Syngenta crop protection, Pakistan and sprayed with Knapsack sprayer P.B-20 (Made by Syngenta crop protection, Malaysia) fitted with hollow cone nozzle.

Harvesting: Harvesting of green pods was started after 4 days of flowering. Pods were picked at the harvestable maturity stage with an interval of 1-2 days and weighed. Picking continued until no economic pods were produced.

Weeds control: Extensive weed infestation was observed in the okra field. Manually seven hand weedings (10, 19, 27, 32, 51, 60 and 72 days after sowing) were done with hand hoeing which were integrated with one chemical control in the first experiment. Dual Gold 960 EC @ $2.0 \mathrm{~L} \mathrm{ha}^{-1}$ (pre-emergence) were sprayed with Knapsack sprayer P.B-20 (Made by 
Syngenta crop protection, Malaysia) fitted with a flat fan nozzle. Major weeds were Cyperusrotundus L., Alternanthera philoxeroides L. (Martius.) and Trianthemaportulac astrum L.

Recordings of weeds, growth and yield data

Sampling technique: Randomly ten plants from each experimental unit were selected and tagged with a label for recording observation on various plant growth and yield characters. Data were recorded from these selected plants, however yield were recorded from the whole experimental unit.

Growth parameters

Days taken to first flower initiation $(D A S)$ : Days to initiation of the first flower were counted from sowing to blooming of the first flower from randomly selected ten plants.

Plant height at flowering (cm): Plant height (cm) was measured from 10 plants and were averaged.

Plant height at maturity $(\mathrm{cm})$ : Plant height $(\mathrm{cm})$ at maturity was measured from 10 selected plants and were averaged.

\section{Yield parameters}

Pod fresh weight (g)/plant: Fresh pods were harvested from 10 randomly selected plants and were weighted and were averaged to get a single pod fresh weight.

Pod length (cm): Fresh pods were randomly harvested at marketable maturity. Pod length $(\mathrm{cm})$ was measured and were averaged.

Pod diameter ( $\mathrm{cm})$ : Pod diameter was measured by using vernier calliper. Five pods from randomly selected plants were harvested. The diameter was measured and then averaged.
Number of pods per plant: Data for number of pods per plant from the total number of pods from tagged plants were counted and an average was calculated.

Pod yield $\left(\boldsymbol{t} \boldsymbol{h a}^{-1}\right)$ : For the determination of pod yield whole plots were harvested. Harvesting of pods commenced when the pods reached at the marketable size. Harvesting of pods continued with one-day interval until when the crop was no longer producing economic pods. The total pod yield recorded here constituted the cumulative harvested pods and was obtained by converting the pod yield $\mathrm{kg}$ per plot to $\mathrm{t} \mathrm{ha}^{-1}$.

\section{Quality analysis}

Pod potassium (\%): Pod potassium (\%) was determined with a flame photometer (Jenwat PFP7) as described by Ryan et al. (2007).

Pod ascorbic acid (\%): Ascorbic acid contents of juice were determined as described by Ruck (1963).

The vitamin $\mathrm{C}$ was calculated by using the formula:

Ascorbic acid (\%)

$=\frac{1 \times \text { dye used against sample } \times \text { sample volume by adding oxalic acid }}{\text { standard reading } \times \text { weight of sample } \times \text { volume of filtrate used }} \times 100$

Pod ash content (\%): Pod ash content was determined with a muffle furnace $(\mathrm{AC}, 1995)$ at $550^{\circ} \mathrm{C}$. The ash contents weredetermined using the formula:

$$
\text { Ash }(\%)=\frac{\text { weight of ash }(\mathrm{g})}{\text { weight of sample }(\mathrm{g})} \times 100
$$

Statistical Analysis: Individual and interactive effects of okra phenotype, growth, yield and quality parameters were analyzed using the Statistix 8.1 statistical computing package. Means were compared at $5 \%$ and $1 \%$ probability. Regression analysis $\left(\mathrm{R}^{2}\right)$ was conducted separately under MS excel for the parameters tested.

Table 3. Effect of nitrogen, phosphorous, potassium (NPK) and Alligator weed compost on days taken to first flower initiation (DAS), plant height (PH) at flowering, plant height (PH) at maturity and pod length (cm) under field conditions

\begin{tabular}{|c|c|c|c|c|c|c|c|c|}
\hline $\begin{array}{l}\text { NPK and alligator weed } \\
\text { compost }\end{array}$ & $\begin{array}{c}\text { Days taken } \\
\text { to } 1^{\text {st flower }} \\
\text { initiation } \\
2017 \\
\end{array}$ & $\begin{array}{c}\text { Days taken } \\
\text { to } 1^{\text {st flower }} \\
\text { initiation } \\
2018 \\
\end{array}$ & 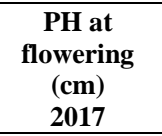 & $\begin{array}{c}\text { PH at } \\
\text { flowering }(\mathrm{cm} \\
) \\
2018 \\
\end{array}$ & $\begin{array}{c}\text { PH at } \\
\text { maturity } \\
(\mathrm{cm}) \\
2017 \\
\end{array}$ & $\begin{array}{c}\text { PH at } \\
\text { maturity } \\
(\mathrm{cm}) \\
2018 \\
\end{array}$ & $\begin{array}{c}\begin{array}{c}\text { Pod length } \\
(\mathrm{cm})\end{array} \\
2017 \\
\end{array}$ & $\begin{array}{c}\begin{array}{c}\text { Pod length } \\
\text { (cm) }\end{array} \\
2018 \\
\end{array}$ \\
\hline $\begin{array}{l}\text { Control (recommended dose } \\
\text { of NPK } 159-114-93 \mathrm{~kg} \mathrm{ha}^{-1} \text { ) }\end{array}$ & $53.75 a \pm 2.1$ & $53.25 \mathrm{a} \pm 4.8$ & $47.79 \mathrm{e} \pm 2.7$ & $52.12 \mathrm{~d} \pm 3.3$ & $71.67 \mathrm{~d} \pm 4.2$ & $74.25 \mathrm{e} \pm 4.6$ & $10.14 \mathrm{c} \pm 0.51$ & $7.45 \mathrm{e} \pm 0.54$ \\
\hline Compost alone $\left(250 \mathrm{~kg} \mathrm{ha}^{-1}\right)$ & $52.50 \mathrm{~b} \pm 4.7$ & $53.00 \mathrm{a} \pm 4.7$ & $54.13 \mathrm{~d} \pm 3.0$ & $52.79 \mathrm{~d} \pm 3.0$ & $75.05 \mathrm{~d} \pm 4.4$ & $74.96 \mathrm{e} \pm 4.7$ & $10.52 b \pm 0.47$ & $7.83 \mathrm{~d} \pm 0.38$ \\
\hline Compost alone $\left(500 \mathrm{~kg} \mathrm{ha}^{-1}\right)$ & $52.50 \mathrm{~b} \pm 4.5$ & $52.50 \mathrm{ab} \pm 4.5$ & $54.83 \mathrm{~cd} \pm 2.8$ & $55.37 \mathrm{c} \pm 3.0$ & $75.63 \mathrm{~d} \pm 4.3$ & $78.12 \mathrm{~d} \pm 4.9$ & $10.57 b \pm 0.49$ & $7.93 \mathrm{~cd} \pm 0.41$ \\
\hline $\begin{array}{l}\text { Compost } 250 \mathrm{~kg} \mathrm{ha}^{-1}+\mathrm{NPK} \\
75 \% \text { of recommended }\end{array}$ & $51.85 b c \pm 3.9$ & $51.75 b \pm 4.3$ & $59.42 \mathrm{a} \pm 3.5$ & $58.54 \mathrm{~b} \pm 3.8$ & $85.08 \mathrm{~b} \pm 5.1$ & $82.29 b \pm 5.3$ & $10.76 b \pm 0.56$ & $8.13 c \pm 0.39$ \\
\hline $\begin{array}{l}\text { Compost } 250 \mathrm{~kg} \mathrm{ha}^{-1}+\mathrm{NPK} \\
50 \% \text { of recommended }\end{array}$ & $52.00 \mathrm{bc} \pm 4.1$ & $52.00 \mathrm{ab} \pm 4.8$ & $58.33 \mathrm{ab} \pm 3.4$ & $58.50 \mathrm{~b} \pm 3.3$ & $84.15 b \pm 5.2$ & $81.95 b c \pm 5.0$ & $10.73 b \pm 0.54$ & $8.12 c \pm 0.42$ \\
\hline $\begin{array}{l}\text { Compost } 250 \mathrm{~kg} \mathrm{ha}^{-1}+\mathrm{NPK} \\
25 \% \text { of recommended }\end{array}$ & $52.25 b c \pm 4.6$ & $52.00 \mathrm{ab} \pm 4.9$ & $55.96 c \pm 3.3$ & $56.75 c \pm 2.4$ & $78.15 c \pm 4.3$ & $78.58 \mathrm{c} \pm 4.8$ & $10.69 b \pm 0.48$ & $8.05 c \pm 0.43$ \\
\hline $\begin{array}{l}\text { Compost } 500 \mathrm{~kg} \mathrm{ha}^{-1}+\mathrm{NPK} \\
75 \% \text { of recommended }\end{array}$ & $51.00 c \pm 3.8$ & $51.25 b c \pm 4.5$ & $60.75 \mathrm{a} \pm 3.5$ & $59.87 \mathrm{~b} \pm 4.2$ & $87.83 \mathrm{a} \pm 5.0$ & $84.54 b \pm 5.8$ & $10.69 b \pm 0.50$ & $8.64 b \pm 0.46$ \\
\hline $\begin{array}{l}\text { Compost } 500 \mathrm{~kg} \mathrm{ha}^{-1}+\mathrm{NPK} \\
50 \% \text { of recommended }\end{array}$ & $51.45 c \pm 3.9$ & $51.50 b c \pm 4.1$ & $59.58 \mathrm{a} \pm 3.0$ & $62.33 \mathrm{a} \pm 4.3$ & $87.63 \mathrm{a} \pm 5.2$ & $92.62 \mathrm{a} \pm 6.1$ & $11.20 \mathrm{a} \pm 0.62$ & $9.14 \mathrm{a} \pm 0.48$ \\
\hline $\begin{array}{l}\text { Compost } 500 \mathrm{~kg} \mathrm{ha}^{-1}+\mathrm{NPK} \\
25 \% \text { of recommended }\end{array}$ & $52.00 \mathrm{bc} \pm 4.4$ & $51.50 b c \pm 4.2$ & $56.71 c \pm 2.9$ & $58.99 \mathrm{~b} \pm 4.0$ & $79.75 c \pm 4.8$ & $83.79 b \pm 5.3$ & $10.72 b \pm 0.52$ & $8.51 b \pm 0.49$ \\
\hline LSD & 1.20 & 1.27 & 1.50 & 1.65 & 2.71 & 2.56 & 0.35 & 0.19 \\
\hline
\end{tabular}




\section{RESULTS}

The combined application of alligator weed compost together with nitrogen, phosphorous and potassium (500 kg ha $^{-1}$ compost $+50 \%$ recommended NPK) significantly improved phenology $(P<0.05)$ and okra plant growth $(P<0.05)$ during both years of study (Table 3$)$. Application of compost at $500 \mathrm{~kg} \mathrm{ha}^{-1}$ along with NPK $50 \%$ of recommended dose decreased days to flowering (2 days) over control (recommended dose of NPK). Plant height (at flowering and at maturity was increased by $23 \%$ and $24 \%$, respectively, as compared to control over both years of study.

Tables 4 and 5 showed that the combined application of compost and NPK significantly increased the length of the pod $(\mathrm{cm})$, the diameter of the pod, the fresh weight of the pod $(\mathrm{g})$, number of pods per plant, pod length and yield of the pod

Table 4. Effect of nitrogen, phosphorous, potassium (NPK) and alligator weed compost on pod diameter, pod fresh weight, number of pods per plant under field conditions

\begin{tabular}{|c|c|c|c|c|c|c|}
\hline $\begin{array}{l}\text { NPK and alligator weed } \\
\text { compost }\end{array}$ & $\begin{array}{c}\text { Pod diameter } \\
\text { (cm) } \\
2017\end{array}$ & $\begin{array}{c}\text { Pod diameter } \\
(\mathrm{cm}) \\
2018 \\
\end{array}$ & $\begin{array}{c}\text { Pod fresh } \\
\text { weight (g) } \\
2017\end{array}$ & $\begin{array}{c}\text { Pod fresh } \\
\text { weight (g) } \\
2018\end{array}$ & $\begin{array}{c}\text { Number of } \\
\text { pods per plant } \\
2017 \\
\end{array}$ & $\begin{array}{c}\text { Number of } \\
\text { pods per plant } \\
2018\end{array}$ \\
\hline $\begin{array}{l}\text { Control (recommended dose } \\
\text { of NPK } 159-114-93 \mathrm{~kg} \mathrm{ha}^{-1} \text { ) }\end{array}$ & $1.38 b c \pm 0.17$ & $1.38 \mathrm{f} \pm 0.16$ & $11.24 \mathrm{e} \pm 0.58$ & $13.02 \mathrm{~cd} \pm 0.66$ & $6.7 \mathrm{~d} \pm 0.40$ & $5.9 \mathrm{f} \pm 0.31$ \\
\hline Compost alone $\left(250 \mathrm{~kg} \mathrm{ha}^{-1}\right)$ & $1.40 \mathrm{bc} \pm 0.17$ & $1.45 \mathrm{e} \pm 0.16$ & $12.25 \mathrm{~d} \pm 0.63$ & $13.25 b c \pm 0.69$ & $7.1 b c \pm 0.44$ & $6.4 \mathrm{de} \pm 0.30$ \\
\hline Compost alone $\left(500 \mathrm{~kg} \mathrm{ha}^{-1}\right)$ & $1.44 b \pm 0.17$ & $1.48 \mathrm{e} \pm 0.17$ & $12.40 \mathrm{~cd} \pm 0.75$ & $13.30 \mathrm{~b} \pm 0.72$ & $7.2 b c \pm 0.43$ & $6.6 \mathrm{~d} \pm 0.33$ \\
\hline $\begin{array}{l}\text { Compost } 250 \mathrm{~kg} \mathrm{ha}^{-1}+\mathrm{NPK} \\
75 \% \text { of recommended }\end{array}$ & $1.50 \mathrm{~b} \pm 0.17$ & $1.63 b \pm 0.18$ & $13.13 \mathrm{c} \pm 0.76$ & $13.69 \mathrm{~b} \pm 0.73$ & $8.0 \mathrm{a} \pm 0.46$ & $7.1 \mathrm{c} \pm 0.32$ \\
\hline $\begin{array}{l}\text { Compost } 250 \mathrm{~kg} \mathrm{ha}^{-1}+\mathrm{NPK} \\
50 \% \text { of recommended }\end{array}$ & $1.49 b \pm 0.15$ & $1.60 b c \pm 0.16$ & $12.78 \mathrm{c} \pm 0.70$ & $13.44 \mathrm{~b} \pm 0.71$ & $7.7 b \pm 0.43$ & $6.7 \mathrm{~d} \pm 0.38$ \\
\hline $\begin{array}{l}\text { Compost } 250 \mathrm{~kg} \mathrm{ha}^{-1}+\mathrm{NPK} \\
25 \% \text { of recommended }\end{array}$ & $1.45 b \pm 0.16$ & $1.56 \mathrm{~cd} \pm 0.18$ & $12.49 \mathrm{~d} \pm 0.68$ & $13.36 \mathrm{~b} \pm 0.69$ & $7.4 \mathrm{~b} \pm 0.40$ & $6.6 \mathrm{~d} \pm 0.36$ \\
\hline $\begin{array}{l}\text { Compost } 500 \mathrm{~kg} \mathrm{ha}^{-1}+\mathrm{NPK} \\
75 \% \text { of recommended }\end{array}$ & $1.51 \mathrm{~b} \pm 0.17$ & $1.66 b \pm 0.17$ & $13.78 \mathrm{~b} \pm 0.78$ & $14.41 \mathrm{a} \pm 0.76$ & $8.3 \mathrm{a} \pm 0.42$ & $7.3 b \pm 0.33$ \\
\hline $\begin{array}{l}\text { Compost } 500 \mathrm{~kg} \mathrm{ha}^{-1}+\mathrm{NPK} \\
50 \% \text { of recommended }\end{array}$ & $1.65 \mathrm{a} \pm 0.18$ & $1.77 \mathrm{a} \pm 0.19$ & $14.56 \mathrm{a} \pm 0.75$ & $14.60 \mathrm{a} \pm 0.82$ & $8.3 \mathrm{a} \pm 0.41$ & $7.7 \mathrm{a} \pm 0.39$ \\
\hline $\begin{array}{l}\text { Compost } 500 \mathrm{~kg} \mathrm{ha}^{-1}+\mathrm{NPK} \\
25 \% \text { of recommended }\end{array}$ & $1.45 b \pm 0.16$ & $1.65 b \pm 0.18$ & $12.64 \mathrm{~cd} \pm 0.65$ & $14.23 \mathrm{a} \pm 0.78$ & $7.5 b \pm 0.39$ & $7.3 b \pm 0.35$ \\
\hline LSD & 0.08 & 0.04 & 0.53 & 0.42 & 0.44 & 0.18 \\
\hline
\end{tabular}

Table 5. Effect of nitrogen, phosphorous and potassium (NPK) and alligator weed compost on pod yield, pod potassium, pod ascorbic acid and pod ash content $(\%)$ under field conditions

\begin{tabular}{|c|c|c|c|c|c|c|c|c|}
\hline $\begin{array}{l}\text { NPK and alligator weed } \\
\text { compost }\end{array}$ & $\begin{array}{c}\text { Pod Yield } \\
\left(\mathrm{t} \mathrm{ha}^{-1}\right) \\
2017\end{array}$ & $\begin{array}{c}\text { Pod Yield } \\
\left(\mathrm{t} \mathrm{ha}^{-1}\right) \\
2018\end{array}$ & $\begin{array}{c}\text { Pod } \\
\text { Potassium( } \\
\%) \\
2017\end{array}$ & $\begin{array}{c}\text { Pod } \\
\text { Potassium( } \\
\%) \\
2018\end{array}$ & $\begin{array}{c}\text { Pod } \\
\text { Ascorbic } \\
\text { Acid }(\%) \\
2017\end{array}$ & $\begin{array}{c}\text { Pod } \\
\text { Ascorbic } \\
\text { Acid(\%) } \\
2018\end{array}$ & $\begin{array}{c}\text { Pod Ash } \\
\text { Content }(\%) \\
2017\end{array}$ & $\begin{array}{c}\text { Pod Ash } \\
\text { Content }(\%) \\
2018\end{array}$ \\
\hline $\begin{array}{l}\text { Control (recommended dose } \\
\text { of NPK } 159-114-93 \mathrm{~kg} \mathrm{ha}^{-1} \text { ) }\end{array}$ & $5.73 \mathrm{~cd} \pm 0.27$ & $6.03 \mathrm{e} \pm 0.28$ & $1.26 \mathrm{ef} \pm 0.06$ & $1.48 \mathrm{c} \pm 0.09$ & $24.04 \mathrm{c} \pm 2.2$ & $25.22 \mathrm{~d} \pm 2.8$ & $13.01 \mathrm{~b} \pm 1.5$ & $9.13 b \pm 0.51$ \\
\hline Compost alone $\left(250 \mathrm{~kg} \mathrm{ha}^{-1}\right)$ & $5.81 \mathrm{c} \pm 0.28$ & $6.21 \mathrm{~d} \pm 0.30$ & $1.44 \mathrm{e} \pm 0.08$ & $1.68 b \pm 0.10$ & $23.42 \mathrm{c} \pm 2.5$ & $27.75 \mathrm{c} \pm 3.0$ & $13.13 b \pm 1.6$ & $9.20 \mathrm{~b} \pm 0.53$ \\
\hline Compost alone (500 $\left.\mathrm{kg} \mathrm{ha}^{-1}\right)$ & $5.95 c \pm 0.29$ & $6.38 c \pm 0.32$ & $1.77 \mathrm{~cd} \pm 0.09$ & $1.71 b \pm 0.10$ & $22.44 c \pm 2.3$ & $28.76 c \pm 2.9$ & $13.57 \mathrm{~b} \pm 1.4$ & $9.32 b \pm 0.51$ \\
\hline $\begin{array}{l}\text { Compost } 250 \mathrm{~kg} \mathrm{ha}^{-1}+\mathrm{NPK} \\
75 \% \text { of recommended }\end{array}$ & $6.47 \mathrm{~b} \pm 0.35$ & $6.53 b \pm 0.33$ & $2.12 \mathrm{ab} \pm 0.13$ & $1.83 b \pm 0.12$ & $28.85 b \pm 2.9$ & $31.48 \mathrm{~b} \pm 3.1$ & $13.63 b \pm 1.6$ & $9.68 b \pm 0.52$ \\
\hline $\begin{array}{l}\text { Compost } 250 \mathrm{~kg} \mathrm{ha}^{-1}+\mathrm{NPK} \\
50 \% \text { of recommended }\end{array}$ & $6.44 b \pm 0.36$ & $6.42 b c \pm 0.31$ & $2.09 \mathrm{ab} \pm 0.11$ & $1.82 \mathrm{~b} \pm 0.11$ & $28.85 b \pm 2.8$ & $30.12 b \pm 3.1$ & $13.35 \mathrm{~b} \pm 1.4$ & $9.55 b \pm 0.58$ \\
\hline $\begin{array}{l}\text { Compost } 250 \mathrm{~kg} \mathrm{ha}^{-1}+\mathrm{NPK} \\
25 \% \text { of recommended }\end{array}$ & $6.02 c \pm 0.31$ & $6.38 \mathrm{~d} \pm 0.33$ & $1.60 \mathrm{c} \pm 0.12$ & $1.72 b \pm 0.11$ & $28.85 b \pm 2.7$ & $29.02 b c \pm 3.0$ & $13.31 \mathrm{~b} \pm 1.5$ & $9.35 b \pm 0.56$ \\
\hline $\begin{array}{l}\text { Compost } 500 \mathrm{~kg} \mathrm{ha}^{-1}+\mathrm{NPK} \\
75 \% \text { of recommended }\end{array}$ & $6.99 a \pm 0.37$ & $6.60 b \pm 0.34$ & $2.33 \mathrm{a} \pm 0.15$ & $2.23 \mathrm{a} \pm 0.12$ & $33.65 \mathrm{a} \pm 3.3$ & $32.99 \mathrm{a} \pm 3.4$ & $14.98 \mathrm{a} \pm 1.9$ & $10.68 \mathrm{a} \pm 0.96$ \\
\hline $\begin{array}{l}\text { Compost } 500 \mathrm{~kg} \mathrm{ha}^{-1}+\mathrm{NPK} \\
50 \% \text { of recommended }\end{array}$ & $7.22 \mathrm{a} \pm 0.39$ & $7.01 \mathrm{a} \pm 0.36$ & $2.44 \mathrm{a} \pm 0.14$ & $2.38 \mathrm{a} \pm 0.13$ & $33.65 \mathrm{a} \pm 3.4$ & $32.82 \mathrm{a} \pm 3.3$ & $15.20 \mathrm{a} \pm 1.7$ & $10.98 \mathrm{a} \pm 0.92$ \\
\hline $\begin{array}{l}\text { Compost } 500 \mathrm{~kg} \mathrm{ha}^{-1}+\mathrm{NPK} \\
25 \% \text { of recommended }\end{array}$ & $6.07 c \pm 0.31$ & $6.56 b \pm 0.35$ & $2.01 b c \pm 0.13$ & $1.84 b \pm 0.11$ & $30.45 b \pm 3.2$ & $30.98 b \pm 3.2$ & $13.59 \mathrm{~b} \pm 1.4$ & $9.74 b \pm 0.95$ \\
\hline LSD & 0.27 & 0.11 & 0.30 & 0.18 & 2.45 & 1.30 & 1.23 & 0.91 \\
\hline
\end{tabular}

Values are the means of four replications $(\mathrm{n}=4) \pm \mathrm{SE}$ and variants possessing the same letters are not statistically significant at $P<0.05$. Main factors and interactions are significant at $P<0.05$ and $P<0.01$. 
Tanveer, Asghar, Sarwar, Saleem, Nadeem, Munir, Zafar, Rizwan \& Sarwar

Table 6. Comparison of net field benefit cost ratio (BCR) of okra for various combination of NPK and Alligator weed compost

\begin{tabular}{|c|c|c|c|c|}
\hline NPK and Alligator weed compost & $\begin{array}{c}\text { Gross income } \\
(\text { Rs. ha } \\
\text { (1) }\end{array}$ & $\begin{array}{c}\text { Gross cost } \\
\left(\text { Rs. ha }^{-1}\right)\end{array}$ & $\begin{array}{l}\text { Net field benefit } \\
\left(\text { Rs. ha }^{-1}\right)\end{array}$ & BCR \\
\hline Control (recommended dose of NPK 159-114-93 $\mathrm{kg} \mathrm{ha}^{-1}$ ) & 744900 & 210522.4 & 534377.62 & 2.54 \\
\hline Compost alone $\left(250 \mathrm{~kg} \mathrm{ha}^{-1}\right)$ & 755300 & 177667.2 & 577632.78 & 3.25 \\
\hline Compost alone $\left(500 \mathrm{~kg} \mathrm{ha}^{-1}\right)$ & 773500 & 178583.2 & 594916.78 & 3.33 \\
\hline Compost $250 \mathrm{~kg} \mathrm{ha}^{-1}+\mathrm{NPK} 75 \%$ of recommended & 841100 & 202995.6 & 638104.41 & 3.14 \\
\hline Compost $250 \mathrm{~kg} \mathrm{ha}^{-1}+$ NPK $50 \%$ of recommended & 837200 & 194552.8 & 642647.20 & 3.30 \\
\hline Compost $250 \mathrm{~kg} \mathrm{ha}^{-1}+$ NPK $25 \%$ of recommended & 782600 & 186110.0 & 596489.99 & 3.21 \\
\hline Compost $500 \mathrm{~kg} \mathrm{ha}^{-1}+$ NPK $75 \%$ of recommended & 908700 & 195468.8 & 713231.20 & 3.65 \\
\hline Compost $500 \mathrm{~kg} \mathrm{ha}^{-1}+\mathrm{NPK} 50 \%$ of recommended & 938600 & 203911.6 & 734688.41 & 3.60 \\
\hline Compost $500 \mathrm{~kg} \mathrm{ha}^{-1}+$ NPK $25 \%$ of recommended & 789100 & 187026.0 & 602073.99 & 3.22 \\
\hline
\end{tabular}

$\left(\right.$ tha $\left.^{-1}\right)$ over the alone application of recommended NPK $(P<0.05, P<0.01)$ during both years of study. The use of compost at $500 \mathrm{~kg} \mathrm{ha}^{-1}$ along with NPK at $50 \%$ of the recommended dose increased the number of pods per plant and pod yield by $26 \%, 20 \%$, respectively averaged across during both years of study over the check treatment. Similarly, averaged across during both years of study, the pod fresh weight $(\mathrm{g})$ was increased by $21 \%$ when combined compost $\left(500 \mathrm{~kg} \mathrm{ha}^{-1}\right)$ and NPK (50\% of the recommended dose) were applied whencompared to the recommended NPK application alone. The data in Table 5 showed that the okra pod potassium contents (\%), ascorbic acid contents (\%) and ash contents (\%) were significantly improved under integrated application of compost and NPK when compared to NPK application alone in both years $(P<0.05, P<0.01)$. Applying compost at $500 \mathrm{~kg} \mathrm{ha}^{-1}$ along with NPK at $50 \%$ of the prescribed dose increased pod potassium and ascorbic acid contents by $76 \%$ and $35 \%$, respectively, during the two years of study over the NPK control dose. Similar to the pod potassium and ascorbic acid contents, combined application of compost and NPK also increased pod ash contents by $17 \%$ over the control. The application of compost at $500 \mathrm{~kg} \mathrm{ha}^{-1}$ together with NPK at $75 \%$ of the recommended dose produced similar results for phenotype, growth, yield and yield related components and okra pod quality components as represented by the application of compost at $500 \mathrm{~kg} \mathrm{ha}^{-1}$ along with NPK at $50 \%$ of recommended dose. Economic analysis of the different combinations of compost with different NPK levels in Table 6 showed that the application of compost at $500 \mathrm{~kg} \mathrm{ha}^{-1}$ along with NPK $50 \%$ of the recommended dose was the most cost-effective treatment that resulted in the highest net field gain and cost-benefit ratio. The application of compost at500 $\mathrm{kg} \mathrm{ha}^{-1}$ together with NPK $75 \%$ of the recommended dose produced a similar cost-benefit ratio.

Relationship between pod yield, yield components and pod quality components: The relation between the yield of the pod and the yield contribution components [no. of pods per plant, pod length $(\mathrm{cm})$, pod diameter $(\mathrm{cm})$, and pod fresh weight (g)] (Fig. 2a) and pod yield with pod quality components [pod potassium content (\%), pod ascorbic acid content (\%) and pod ash content (\%) (Fig. 2b) was analyzed through regression analysis. Pod yield showed a positive and significantly higher relationship with yield and quality components. Mean regression squares were significant at $1 \%$ when analyzed in the t-test analysis.

(a)

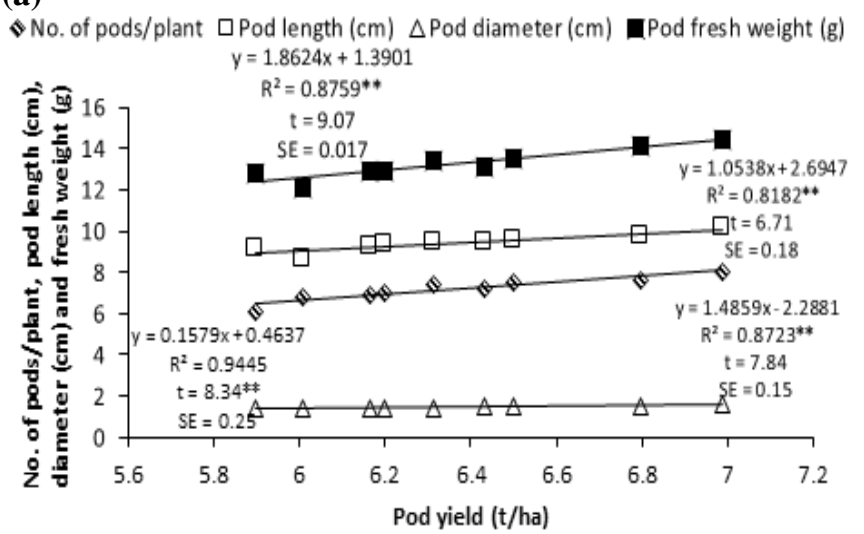

(b)

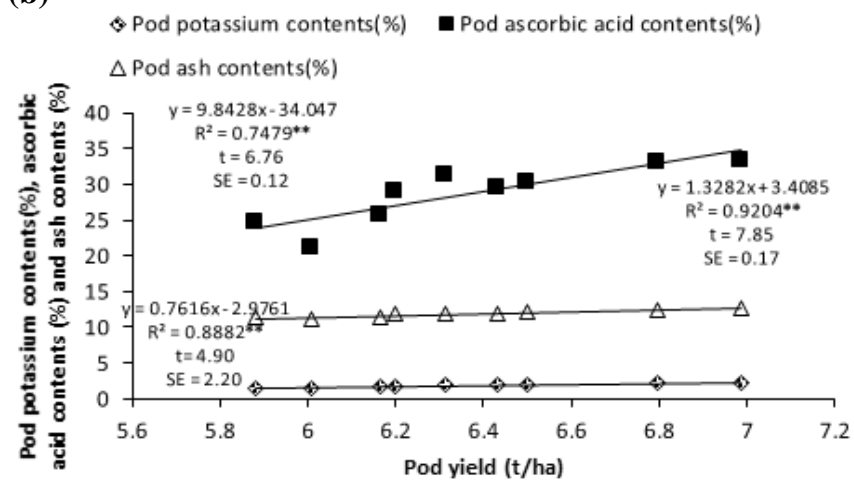

Figure 2. (a) Association between pod yield (t/ha) with no. of pods per plant, pod length $(\mathrm{cm})$, pod diameter (cm) and pod fresh weight (b) Association between pod yield (t/ha) with pod potassium contents (\%), pods ascorbic acid contents (\%) and pod ash contents $(\%)$. 


\section{DISCUSSION}

The use of compost along with nutrients improves the quality of fertilizer use. The use of nutrients with compost slows down the release of nutrients rather than rapidly making them available. It maintains optimal plant growth (Setyowati et al., 2017) and improves the crop phenology needed for early crop maturity. Similar data have been proposed by Prabu et al. (2002), which found that the combined use of compost and inorganic fertilizers enhances crop phenology. Emma-Okafor et al. (2017) stated that the use of compost in plant contributes to a favorable growth environment that improves flowering by improving soil physio-chemical properties. Integrated use of compost and inorganic fertilizers creates favorable conditions for proper growth and development; increases cell division and cell elongation; improves root development; improves the uptake of water and nutrients which improves plant height (Barani and Anburani, 2004). In the present study, plant height was increased at flowering and maturity when compost and inorganic fertilizers $(75 \%$ of recommended NPK) were applied together. This may be due to an increase in the availability of nutrients and the preponderance of different groups of microorganisms present in compost as well as in soil, which provide a favorable environment for proper vegetative growth in general and increased plant height in particular. Similar findings have been suggested by Varma and Kuldeep (2003) in peas (Pisum sativum L.) Patil (2010) in stevia (Stevia rebaudiana L.), Nyangani (2010) in different field crops and Olusegun (2014) in cow peas (Vigna unguiculata L.) due to the combined use of organic and inorganic fertilizers. Every crop requires nutrients for its growth and development, but the supply of nutrients for each crop is different. Applying nutrients to crops in a sustainable manner is better and less costly. Composting is a cost-effective way, composting material enriches soil, helps to retain moisture, promotes the growth of beneficial bacteria, decreases the need for chemical fertilizers and increases crop production (Wright et al., 2007; Jouquet et al., 2011). Dorahy et al. (2009) reported that composting is an effective technique for reducing aquatic and terrestrial weeds. The preparation of compost from aquatic weeds is an environmental and cost-effective technique for the preparation of organic material. In this study, the use of compost with the half-dose of recommended NPK improved the length of the pod $(\mathrm{cm})$, the diameter of the pod $(\mathrm{cm})$, the fresh weight of the pod (g), the number of pods per plant and the yield of the pod $\left(\right.$ tha $\left.^{-1}\right)$ which may be due to the combination of compost and inorganic fertilizers which increased the production of photosynthesis and the partitioning of dry matter at the growth stage. These findings are in agreement with the findings of some researchers (Abd El-Kader et al., 2010; Afe and Oluleye, 2017) and other crops such as maize (Kolawole, 2014), sorghum (Bayu et al., 2006), rice (Satyanarayana et al., 2007), sweet maize (Uwah et al.,
2011), cowpea (Olusegun, 2014), chili pepper (Setyowati et al., 2017) and plantain (Ekpo and Ekpo, 2019). Potassium has an important role in growth, photosynthesis, biotic/abiotic stress relief, ascorbic acid production and sucrose translocation (Lester et al., 2005; Hawkesford, 2014). Ascorbic acid is the most abundant and effective antioxidant in the plant body, retains redox potential within cells, enhances cell vision and photosynthesis (Smirnoff and Wheeler, 2000; Zhang, 2012). Quality evaluation of the okra used to be done based on calcium, potassium, ascorbic acid and fiber contents. Okra serves as a tonic for both men and women, helping them to increase their strength and vigor (Pendre et al., 2012). The potassium and ascorbic acid content of okra pods have increased significantly as a result of the combined use of compost and inorganic fertilizers, which may be due to the high availability of potassium from the adsorption sites of alligator weed compost during pod formation. High potassium availability may also increase the development of ascorbic acid content by triggering the plant defensive system. These findings have been supported by Adekiya and Agbede(2009) who found that the combined use of organic and inorganic fertilizers increased the potassium contents of tomato leaves. Similarly, Hassan et al. (2012) suggested that organic based treatments/fertilizers improve ascorbic acid contents in Cosmos caudatus when compared with inorganic fertilizers. Zodape et al. (2011) suggested that the application of different organic fertilizers can influence the nutritional quality of tomato as found in this study where the combined use of organic and inorganic fertilizers improved the fiber contents in okra pods as found in tomato by Ilupeju et al. (2015). Okra pod yield has a positive and strong relationship with yield contribution components as indicated by Balakrishnan and Sreenivasan (2010) which may be due to the combined use of organic and inorganic fertilizers, improving quality components along with yield components.

Conclusions: The use of compost with half the recommended dose of NPK gives a higher yield of okra than compost alone or the recommended dose of NPK alone. The result shows that alligator weed compost can replace inorganic fertilizers. This work lacks knowledge of the microbial population of soils used for compost. In the future, soils that are influenced by salt and heavy metals, compost would be tested on these soils to see its role in soil texture, structural improvement and crop yield.

Acknowledgment: This study was financially supported by Pakistan Science Foundation (PSF) project No. PSF/Res/PAU/Agr (467). 


\section{REFERENCES}

Abd El-Kader, A., S. Shaaban and M. Abd El-Fattah. 2010. Effect of irrigation levels and organic compost on okra plants (Abelmoschus esculentus L.) grown in sandy calcareous soil. Agric. Biol. J. North Am. 1:255-231.

AC, A. 1995. Official methods of analysis $16^{\text {th }}$ edition. Association of official analytical chemists. Washington, DC, USA.

Adekiya, A. and T. Agbede. 2009. Growth and yield of tomato (Lycopersicon esculentum Mill) as influenced by poultry manure and NPK fertilizer. Emirates J. Food Agri. 21:10-20.

Afe, A. and F. Oluleye. 2017. Response of okra (Abelmuschus esculenthus L. Moench) to combined organic and inorganic foliar fertilizers. Int. J. Recycl. Org. Waste Agri. 6:189-193.

Akande, M., F. Oluwatoyinbo, E. Makinde, A. Adepoju and I. Adepoju. 2010. Response of okra to organic and inorganic fertilization. Nat. Sci. 8:261-266.

Aulakh, M. and C.A. Grant. 2008. Integrated nutrient management for sustainable crop production. CRC Press.

Balakrishnan, D. and E. Sreenivasan. 2010. Correlation and path analysis studies in okra Abelmoschus esculentus $(\mathrm{L}$.) Moench. Madras Agric. J. 97:326-328.

Barani, P. and A. Anburani. 2004. Influence of vermicomposting on major nutrients in bhendi (Abeimoschus esculentus L. Moench) var. Arka Anamika. South Ind. Hort. 52:170.

Bayu, W., N. Rethman, P. Hammes and G. Alemu. 2006. Effects of farmyard manure and inorganic fertilizers on sorghum growth, yield, and nitrogen use in a semi-arid area of Ethiopia. J. Plant Nutr. 29:391-407.

Brown, S. and M. Cotton. 2011. Changes in soil properties and carbon content following compost application: results of on-farm sampling. Compost Sci. Util. 19:8796.

Dorahy, C., A. Pirie, I. McMaster, L. Muirhead, P. Pengelly, K. Chan, M. Jackson and I. Barchia. 2009. Environmental risk assessment of compost prepared from Salvinia, Egeria densa, and Alligator Weed. J. Environ. Quality. 38:1483-1492.

Ekpo, T. and N. Ekpo. 2019. Effects of weed management system and compost manure on plantain yield in agricultural research farm, AfahaNsit, Akwalbom State, Nigeria. J. Appl. Sci. Environ. Manage. 23:1643-1648.

Emma-Okafor, L.C., J.C. Obiefuna, U.P. Iwuanyanwu, N.A. Okoli, I.I. Ibeawuchi and R.A. Alagba.2017. Organic manure for sustainable productivity of plantain/cassava mixture in the tropical ultisol of south-eastern nigeria. Paper presented at the national annual conference organized by Crop Sci. Soc. Nig. (CSSN) held at University of Uyo, Nig. Sept. 10-14.
Fortuna, A., P. Rieke, L. Jacobs, B. Leinauer and D. Karcher. 2005. Kentucky bluegrass response to use of aquatic plants as a soil amendment. Hort. Sci. 40:237-241.

Hansson, P.-A. and H. Fredriksson. 2004. Use of summer harvested common reed (Phragmites australis) as nutrient source for organic crop production in Sweden. Agric. Ecosyst. Environ. 102:365-375.

Hassan, S.A., S. Mijin, U.K. Yusoff, P. Ding and P.E.M. Wahab. 2012. Nitrate, ascorbic acid, mineral and antioxidant activities of Cosmos caudatus in response to organic and mineral-based fertilizer rates. Mol. 17:78437853.

Hawkesford, M. 2014.Functions of macronutrients. University of Copenhagen.

Ilupeju, E., W. Akanbi, J. Olaniyi, B. Lawal, M. Ojo and P. Akintokun. 2015. Impact of organic and inorganic fertilizers on growth, fruit yield, nutritional and lycopene contents of three varieties of tomato (Lycopersicon esculentum (L.) Mill) in Ogbomoso, Nigeria. Afr. J. Biotechnol. 14:2424-2433.

Jouquet, E., E. Bloquel, T.T. Doan, M. Ricoy, D. Orange, C. Rumpel and T.T. Duc. 2011. Do compost and vermicompost improve macronutrient retention and plant growth in degraded tropical soils? Compost Sci. Util. 19:15-24.

Julien, M.H., B. Skarratt and G. Maywald. 1995. Potential geographical distribution of alligator weed and its biological control by Agasicles hygrophila. J. Aquat. Plant Manage. 33:55-60.

Kolawole, O. 2014. Composted municipal solid waste and NPK fertilizer effect on yield, yield components and proximate composition of maize. Afr. J. Agric. Res. 9:1778-1786.

Lester, G.E., J.L. Jifon and G. Rogers. 2005. Supplemental foliar potassium applications during muskmelon fruit development can improve fruit quality, ascorbic acid, and beta-carotene contents. J. Am. Soc. Hortic. Sci. 130:649653.

Manral, H. and S. Saxena. 2003. Plant growth, yield attributes and grain yield of soyabean as affected by the application of inorganic and organic sources of nutrients. Bioresour. Technol. 92:110-118.

Nyangani, E.T. 2010. Effect of combined application of organic manure and chemical fertilizers on soil properties and crop yields: A review. Nig. J. Sci. Tech. Environ. Edu. 3:28-32.

Olusegun, O.S. 2014. Influence of NPK 15-15-15 fertilizer and pig manure on nutrient dynamics and production of cowpea, Vigna unguiculata L. Walp. A.J. Agri.For. 2:267-273.

Oyewole, C.I., S. Amhakhian and O. Saliu. 2011. Response of tomato (Lycopersicon esculentum) and okra (Abelmoschus esculentus (L.) Moench) to rates of NPK nutrients applied as mineral, poultry manure and oil palm 
residue in the guinea savanna agro-ecological zone in Nigeria. J. Int. Sci.Publ: Agri. Food. 2:212-218.

Patil, N. 2010. Biofertilizer effect on growth, protein and carbohydrate content in Stevia rebaudiana var Bertoni.Recent Res. Sci. Technol. 2:42-44.

Pendre, N., P.K. Nema, H.P. Sharma, S. Rathore and S. Kushwah. 2012. Effect of drying temperature and slice size on quality of dried okra (Abelmoschus esculentus (L.) Moench). J. Food Sci. Technol. 49:378-381.

Prabu, T., P. Narwadkar and A. Sajindranath. 2002. Economics of integrated nutrient management in okra. J.Maharashtra Agric. Univ. (Ind). 27:316-318.

Ruck, J. 1963. Chemical methods for analysis of fruit and vegetable products. Contribution no. B7, Research Station, Summerland B.C. 50. Series: Publication. 1154. Ottawa:Canada Department of Agriculture. pp. 16-17. doi: https://doi.org/10.5962/bhl.title.58896.

Ryan, J., G. Estefan and A. Rashid. Soil and Plant Analysis Laboratory Manual. ICARDA.

Sameera, D., V. Shankaraiah and D. Srihari. 2005. Effect of packaging and storage on organic manures grown okra (Abelmoschus esculentus L. Moench). J. Res.Angrau. 33:30-35.

Sarwar, G. 2005. Use of compost for crop production in Pakistan. Univ. Kassel, Fachgebiet Landschaftsökologie und Naturschutz.

Satyanarayana, A., T. Thiyagarajan and N. Uphoff. 2007. Opportunities for water saving with higher yield from the system of rice intensification. Irrig. Sci. 25:99-115.

Savello, P.A., F.W. Martin and J.M. Hill. 1980. Nutritional composition of okra seed meal. J. Agric. Food Chem. 28:1163-1166.

Setyowati, N., Z. Muktamar, B. Suriyanti and M. Simarmata. 2017. Growth and yield of chili pepper as affected by weedbased organic compost and nitrogen fertilizer.doi:10.31219/osf.io/yxm3c.

Smiciklas, K., P. Walker and T. Kelley. 2008. Evaluation of compost for use as a soil amendment in corn and soybean production. Compost Sci.Util. 16:183-191.

Smirnoff, N. and G.L. Wheeler. 2000. Ascorbic acid in plants: biosynthesis and function. Crit. Rev. Biochem. Mol. Biol. 35:291-314.

Tiamiyu, R., H. Ahmed and A. Muhammad. 2012. Effect of sources of organic manure on growth and yields of okra (Abelmoschus esculentus L.) in Sokoto, Nigeria. Niger. J. Basic Appl. Sci. 20:213-216.

Uwah, D., A. Eneji and U. Eshiet. 2011. Organic and mineral fertilizers effects on the performance of sweet maize (Zea mays L.) in southeastern rainforest zone of Nigeria. Int. J. Agri. Sci. 3:54.

Varma, K.S. 2003. Standardization of potassium fertilizer application technology on seed production of pea cultivar arkel. M.Sc. diss., College Horti. Uni. Hort and Forestry, Solan.

Wright, A.L., T.L. Provin, F.M. Hons, D.A. Zuberer and R.H. White. 2007. Compost source and rate effects on soil macronutrient availability under saint augustine grass and bermuda grass turf. Compost Sci.Util. 15:22-28.

Zhang, Y. 2012. Biological role of ascorbate in plants. In: Ascorbic acid in plants: biosynthesis, regulation and enhancement. Springer Heidelberg Dordrecht, London. pp. 7-23

Zodape, S., A. Gupta, S. Bhandari, U. Rawat, D. Chaudhary, K. Eswaran and J. Chikara. 2011. Foliar application of seaweed sap as biostimulant for enhancement of yieldand quality of tomato (Lycopersicon esculentum Mill.). J. Sci. Ind. Res. 70:215-219. 